\title{
Quantum-Liouville and Langevin Equations for Gravitational Radiation Damping
}

\author{
Z. Haba* and H. Kleinert ${ }^{\dagger}$ \\ Institut für Theoretische Physik, \\ Freie Universität Berlin, Arnimallee 14, 14195 Berlin, Germany
}

\begin{abstract}
From a forward-backward path integral, we derive a master equation for the emission and absorption of gravitons by a massive quantum object in a heat bath of gravitons. Such an equation could describe collapse phenomena of dense stars. We also present a useful approximate Langevin equation for such a system.
\end{abstract}

\section{INTRODUCTION}

Usually, quantum effects are negligible for objects of a large mass which consist of a great number of particles. Under special conditions, however, such objects can be of microscopic size. Then the weak gravitational forces may sum up coherently. The process of a collapse of a star will be accompanied by an emission of gravitational waves which should be treated quantum mechanically in the final stages, e.g. during the formation of primordial black holes. In this paper we set up an equation for describing the behavior of a gravitational object of this kind when interacting with an equilibrium state of gravitons.

\section{PATH INTEGRAL FOR DENSITY MATRIX}

Consider the Schrödinger equation

$$
i \hbar \partial_{t}\langle\mathbf{x} \mid \psi(t)\rangle=\hat{H}(t)\langle\mathbf{x} \mid \psi(t)\rangle,
$$

where $\hat{H}(t)=-\hbar^{2} \Delta / 2 M+V(\mathbf{x})$ is the Hamilton operator formed from the Laplace-Beltrami operator $\Delta=$ $g^{-1 / 2}\left(\partial_{\mu} g^{\mu \nu} g^{1 / 2} \partial_{\nu}\right)$ containing the inverse $g^{\mu \nu}$ of the metric $g_{\mu \nu}(\mathbf{x}, t)$ and the determinant $g=\operatorname{det}\left(g_{\mu \nu}\right)$. In empty space, small fluctuations $2 \varepsilon u_{\mu \nu}(x)$ of the metric around the Minkowski metric $\eta_{\mu \nu}$ describe gravitational waves which may be quantized as usual. In the linear approximation, the Einstein action reads

$$
\mathcal{A}_{\mathrm{E}}=-\frac{1}{2 \varepsilon^{2}} \int d^{4} x \sqrt{-g} R=-\frac{1}{2} \int d^{4} x G^{\mu \nu} u_{\mu \nu}, \quad G^{\mu \nu} \equiv \epsilon_{\mu \lambda \sigma \tau} \epsilon_{\nu \lambda \kappa \delta} \partial_{\sigma} \partial_{\kappa} u_{\tau \delta},
$$

where $G^{\mu \nu}$ is the linearized Einstein tensor $R^{\mu \nu}-g^{\mu \nu} R / 2$, and $\varepsilon$ a gravitational coupling constant related to Newton's gravitational constant $\varepsilon^{2}=8 \pi G / c^{3}$. In the radiation gauge with $u^{0 a} \equiv 0$, the free fields may be expanded into creation and annihilation operators for gravitons as

$$
\hat{u}_{\mu \nu}(x)=\sum_{\mathbf{k}} \sum_{h=-2,2} \frac{1}{\sqrt{2 V \Omega_{\mathbf{k}}}}\left[\epsilon_{\mu \nu}(\hat{\mathbf{k}}, h) \hat{a}(\hat{\mathbf{k}}, h) e^{-i k x}+\text { h.c. }\right]
$$

where $\epsilon_{\mu \nu}(\hat{\mathbf{k}}, \pm 2)$ are the traceless divergence-free polarization tensors of helicity \pm 2 . The correlation functions at temperature $T$ of these fields are:

$$
\left\langle\hat{T} \hat{u}_{i j}(\mathbf{x}, t) \hat{u}_{k l}\left(\mathbf{x}^{\prime}, t^{\prime}\right)\right\rangle \equiv G_{i j ; k l}\left(x, x^{\prime}\right)=c \hbar \int \frac{d^{3} k}{(2 \pi)^{3} \Omega_{\mathbf{k}}} \Lambda_{i j ; k l}(\hat{\mathbf{k}}) \cos \mathbf{k}\left(\mathbf{x}-\mathbf{x}^{\prime}\right)\left[\cos \Omega_{\mathbf{k}}\left(t-t^{\prime}\right) \operatorname{coth} \frac{\beta \hbar \Omega_{\mathbf{k}}}{2}-i \sin \Omega_{\mathbf{k}}\left(t-t^{\prime}\right)\right]
$$

where $\Omega_{k}=c|\mathbf{k}|$ and

$$
\Lambda_{i j, k l}(\hat{\mathbf{k}}) \equiv \sum_{h=-2,2} \epsilon(\hat{\mathbf{k}}, h) \epsilon(\hat{\mathbf{k}}, h)^{*}=\frac{1}{2}\left[\Lambda_{i k}(\hat{\mathbf{k}}) \Lambda_{j l}(\hat{\mathbf{k}})+\Lambda_{i l}(\hat{\mathbf{k}}) \Lambda_{j k}(\hat{\mathbf{k}})-\Lambda_{i j}(\hat{\mathbf{k}}) \Lambda_{k l}(\hat{\mathbf{k}})\right]
$$

*On leave from Institute of Theoretical Physics, University of Wroclaw, Poland; e-mail: zhab@ift.uni.wroc.pl

${ }^{\dagger}$ Email: kleinert@physik.fu-berlin.de URL: http://www.physik.fu-berlin.de/ $k$ kleinert 
is the projection tensor to the physical polarization states, expressed in terms of transverse projection matrices $\Lambda_{i j}(\hat{\mathbf{k}})=\delta_{i j}-k_{i} k_{j} / \mathbf{k}^{2}$ of electromagnetism. It is easy to verify the projection property: $\Lambda_{i j, k l}(\hat{\mathbf{k}}) \Lambda_{m n}^{k l}(\hat{\mathbf{k}})=\Lambda_{i j, m n}(\hat{\mathbf{k}})$. As usual, $\beta \equiv 1 / k_{B} T$, and $k_{B}$ is the Boltzmann constant. We have used the four-vector notation $x \equiv(\mathbf{x}, t)$ for space and time. The operator $\hat{T}$ is the time-ordering operator.

We solve the Schrödinger equation (2.1) with the initial condition $\langle\mathbf{x} \mid \psi(0)\rangle=\psi(\mathbf{x})$ and average over the gravitons. As a result we obtain a density matrix

$$
\rho\left(\mathbf{x}_{+}, \mathbf{x}_{-} ; t\right)=\left\langle\mathbf{x}_{+} \mid \psi(t)\right\rangle\left\langle\psi(t) \mid \mathbf{x}_{-}\right\rangle
$$

The time evolution of $\rho\left(\mathbf{x}_{+}, \mathbf{x}_{-} ; t\right)$ is described by the evolution equation

$$
\rho\left(\mathbf{x}_{+a}, \mathbf{x}_{-a} ; t_{b}\right)=\int d \mathbf{x}_{+b} d \mathbf{x}_{-b} U\left(\mathbf{x}_{+b} \mathbf{x}_{-b}, t_{b} \mid \mathbf{x}_{+a} \mathbf{x}_{-a}, t_{a}\right) \rho\left(\mathbf{x}_{+b}, \mathbf{x}_{-b}, t_{a}\right) .
$$

With the help of the influence functional of Feynman and Vernon [1] 3], the kernel of this integral equation is expressed by the forward-backward path integral (see [-4, and compare with the electromagnetic case in [5])

$$
\begin{aligned}
& U\left(\mathbf{x}_{+b} \mathbf{x}_{-b}, t_{b} \mid \mathbf{x}_{+a} \mathbf{x}_{-a}, t_{a}\right)= \int \mathcal{D} \mathbf{x}_{+} \mathcal{D} \mathbf{x}_{-} \exp \left\{\frac{i}{\hbar} \int_{t_{a}}^{t_{b}} d t^{\prime}\left[\frac{M}{2}\left[\dot{\mathbf{x}}_{+}^{2}-\dot{\mathbf{x}}_{-}^{2}\right]-V\left(\mathbf{x}_{+}\right)+V\left(\mathbf{x}_{-}\right)\right]\right\} \\
& \times \exp \left[-\frac{\epsilon^{2} M^{2}}{2 \hbar^{2}} \int_{t_{a}}^{t_{b}} d t \int_{t_{a}}^{t} d t^{\prime}\left(\dot{x}_{+}^{k} \dot{x}_{+}^{l}-\dot{x}_{-}^{k} \dot{x}_{-}^{l}\right) A_{k l ; m n}\left(\dot{x}_{+}^{\prime m} \dot{x}_{+}^{\prime n}-\dot{x}_{-}^{\prime} \dot{x}_{-}^{\prime} n\right)\right. \\
& \\
&\left.\quad-\frac{\epsilon^{2} M^{2}}{2 \hbar^{2}} \int_{t_{a}}^{t_{b}} d t \int_{t_{a}}^{t} d t^{\prime}\left(\dot{x}_{+}^{k} \dot{x}_{+}^{l}-\dot{x}_{-}^{k} \dot{x}_{-}^{l}\right) C_{k l ; m n}\left(\dot{x}_{+}^{\prime m} \dot{x}_{+}^{\prime}{ }_{+} \dot{x}_{-}^{\prime} \dot{x}_{-}^{\prime n}\right)\right]
\end{aligned}
$$

where $x^{\prime}$ stands for $x\left(t^{\prime}\right)$ and $A_{k l ; m n}$ and $C_{k l ; m n}$ are twice the real and imaginary parts of (2.4). We are assuming the wavelengths to be much large than the size of the object, which allows us to neglect the $\mathrm{x}$-dependence in $A_{k l ; m n}$ and $C_{k l ; m n}[6]$. Then we take the angular average

$$
\left\langle\Lambda_{k l ; m n}(\hat{\mathbf{k}})\right\rangle=\Lambda_{k l ; m n} \equiv \frac{2}{5}\left[\frac{1}{2}\left(\delta_{k m} \delta_{l n}+\delta_{k n} \delta_{l m}\right)-\frac{1}{3} \delta_{k l} \delta_{m n}\right]
$$

and find

$$
C_{k l ; m n} \approx i \frac{\hbar}{2 \pi c^{2}} \Lambda_{k l ; m n} \partial_{t} \delta\left(t-t^{\prime}\right)
$$

and at high temperature:

$$
A_{k l ; m n}=\frac{\hbar}{2 \pi c^{2}} \frac{1}{\beta \hbar} \Lambda_{k l ; m n} \delta\left(t-t^{\prime}\right)
$$

Introducing the traceless dimensionless tensor

$$
q^{k l} \equiv \frac{1}{c^{2}}\left(\dot{x}^{k} \dot{x}^{l}-\frac{1}{3} \delta^{k l} \dot{x}_{i} \dot{x}_{i}\right),
$$

the second exponent can be rewritten as

$$
-i \frac{\gamma}{2} \int_{t_{a}}^{t_{b}} d t\left(q_{+}^{k l}-q_{-}^{k l}\right) \partial_{t}\left(q_{+}^{k l}+q_{-}^{k l}\right)^{R}-\frac{w}{2} \int_{t_{a}}^{t_{b}} d t\left(q_{+}^{k l}-q_{-}^{k l}\right)^{2},
$$

where

$$
\gamma \equiv \frac{\varepsilon^{2} M^{2} c^{2}}{10 \pi \hbar}, \quad w \equiv 2 \gamma \frac{k_{B} T}{\hbar} .
$$

and the superscript $R$ emphasizes the retardedness of the $\dot{q}_{k l}$-terms with respect to the $q_{k l}$-terms. This will be important to fix the operator order in the master equation to be derived below. Note that $\gamma$ is dimensionless, whereas $w$ is a frequency. 


\section{MASTER EQUATION FOR DENSITY MATRIX}

Let us derive an operator differential equation for the time evolution of the density matrix. We denote the Hamilton operator without gravitational field by

$$
\hat{H}=\frac{\hat{\mathbf{p}}^{2}}{2 M}+V(\mathbf{x}),
$$

and proceeding as in the electromagnetic discussion in Ref. [5]. The result is the master equation

$\partial_{t} \hat{\rho}=-\frac{i}{\hbar} \mathcal{H} \hat{\rho} \equiv-\frac{i}{\hbar}[\hat{H}, \hat{\rho}]-i \frac{\gamma}{2}\left(\hat{q}^{k l} \hat{\dot{q}}^{k l} \hat{\rho}-\hat{\rho} \hat{\dot{q}}^{k l} \hat{q}^{k l}+\hat{q}^{k l} \hat{\rho} \hat{\dot{q}}^{k l}-\hat{\dot{q}}^{k l} \hat{\rho} \hat{q}^{k l}\right)-\frac{w}{2}\left\{\left[\hat{q}^{k l},\left[\hat{q}^{k l}, \hat{\rho}\right]\right]+\frac{\beta^{2} \hbar^{2}}{12}\left[\hat{\dot{q}}^{k l},\left[\hat{\dot{q}}^{k l}, \hat{\rho}\right]\right]\right\}$

where $\hat{\dot{x}}^{k}, \hat{\ddot{x}}^{k}, \hat{\dot{q}}^{k l}, \ldots$ are defined recursively by the commutators $\hat{\dot{x}}^{k} \equiv i\left[\hat{\mathcal{H}}, \hat{x}^{k}\right] / \hbar, \quad \hat{\ddot{x}}^{k} \equiv i\left[\hat{\mathcal{H}}, \hat{\dot{x}}^{k}\right] / \hbar, \hat{\dot{q}}^{k l} \equiv$ $i\left[\hat{\mathcal{H}}, \hat{q}^{k l}\right] / \hbar, \ldots$.

This equation will conserve the positivity of the probability as can be assured by bringing it the the extended Lindblad form [7]

$$
\partial_{t} \hat{\rho}=-\frac{i}{\hbar}[\hat{H}, \hat{\rho}]-\sum_{n=1}^{2} \sum_{k l}\left(\frac{1}{2} \hat{L}_{n}^{k l} \hat{L}_{n}^{k l \dagger} \hat{\rho}+\frac{1}{2} \hat{\rho} \hat{L}_{n}^{k l} \hat{L}_{n}^{k l \dagger}-\hat{L}_{n}^{k l \dagger} \hat{\rho} \hat{L}_{n}^{k l}\right)
$$

with the Lindblad operators

$$
\hat{L}_{1}^{k l} \equiv \frac{\sqrt{w}}{2} \hat{q}^{k l}, \quad \hat{L}_{2}^{k l} \equiv \frac{\sqrt{3 w}}{2}\left(\hat{q}^{k l}-i \frac{\hbar}{3 k_{B} T} \hat{\dot{q}}^{k l}\right) .
$$

The master equation (3.2) allows us to calculate the rate of change of an initial eigenstate $|i\rangle$ of the Schrödinger equation $H|i\rangle=E_{i}|i\rangle$ by emission and absorption of gravitons to lowest order in $G$. It is a purely classical rate, determined by the matrix elements of the $\gamma$-terms in (3.2) (see [5] for more details):

$$
\partial_{t} \hat{\rho}_{i}=-\frac{\gamma}{2}\left[\left\langle i\left|\hat{q}_{k l}\right| f\right\rangle\left\langle f\left|\hat{\dot{q}}_{k l}\right| i\right\rangle-\left\langle i\left|\hat{\dot{q}}_{k l}\right| f\right\rangle\left\langle f\left|\hat{q}_{k l}\right| i\right\rangle-\left\langle i\left|\hat{q}_{k l}\right| i\right\rangle\left\langle i\left|\hat{\dot{q}}_{k l}\right| i\right\rangle+\left\langle i\left|\hat{\dot{q}}_{k l}\right| i\right\rangle\left\langle i\left|\hat{q}_{k l}\right| i\right\rangle\right]=-\gamma \sum_{f \neq i} \omega_{i f}\left\langle i\left|\hat{q}_{k l}\right| f\right\rangle\left\langle f\left|\hat{q}_{k l}\right| i\right\rangle .
$$

The part of the sum with $f<i$, where the final energy is lower than the initial energy, yields the spontaneous decay rate of the system:

$$
\Gamma=2 \gamma \sum_{f<i} \omega_{i f}\left\langle i\left|\hat{q}_{k l}\right| f\right\rangle\left\langle f\left|\hat{q}_{k l}\right| i\right\rangle
$$

For more details of this identification, in particular the origin of the factor 2 , see Ref. [5]. This decay rate coincides with the perturbative quantum-mechanical result obtained from Fermi's golden rule and the interaction operator $M^{-1} \int_{t_{q}}^{t_{b}} u_{k l} \hat{p}_{k} \hat{p}_{l}$ :

$$
\Gamma=\frac{\varepsilon^{2}}{8 \pi^{2} \hbar} \frac{1}{c^{2} M^{2}} \int d \Omega \sum_{h=-2,2} \sum_{f<i} \omega_{i f}\left|\epsilon^{k l}(\hat{\mathbf{k}}, h)\left\langle f\left|\hat{p}_{k} \hat{p}_{l}\right| i\right\rangle\right|^{2}=\frac{\varepsilon^{2}}{2 \pi \hbar} \frac{2}{5 c^{2} M^{2}} \sum_{f<i} \omega_{i f}\left|\left\langle f\left|\hat{p}_{k} \hat{p}_{l}-\frac{1}{3} \mathbf{p}^{2} \delta_{k l}\right| i\right\rangle\right|^{2}
$$

In terms of $\Gamma$, the right-hand side of $(3.5)$ can be written as

$$
\partial_{t} \hat{\rho}_{i}=-\Gamma+\gamma \sum_{f<i} \omega_{i f}\left\langle i\left|\hat{q}_{k l}\right| f\right\rangle\left\langle f\left|\hat{q}_{k l}\right| i\right\rangle-\gamma \sum_{f>i} \omega_{i f}\left\langle i\left|\hat{q}_{k l}\right| f\right\rangle\left\langle f\left|\hat{q}_{k l}\right| i\right\rangle .
$$

By spontaneous emission, $\hat{\rho}_{i}$ decays like $e^{-\Gamma t}$. The rest is due to induced emissions and absorptions contributing to the rate of change of $\hat{\rho}_{i}$.

The total level width is increased further by the induced emissions and absorptions proportional to $w$ in (3.2):

$$
\partial_{t} \rho_{i}=-w\left\{\sum_{f}\left\langle i\left|\hat{q}_{k l}\right| f\right\rangle\left\langle f\left|\hat{q}_{k l}\right| i\right\rangle-\left\langle i\left|\hat{q}_{k l}^{2}\right| i\right\rangle^{2}+\frac{\beta^{2} \hbar^{2}}{12}\left[\sum_{f}\left\langle i\left|\hat{\dot{q}}_{k l}\right| f\right\rangle\left\langle f\left|\hat{\dot{q}}_{k l}\right| i\right\rangle-\left\langle i\left|\hat{\dot{q}}_{k l}^{2}\right| i\right\rangle^{2}\right]\right\} .
$$


These are due to further induced emission and absorption processes (see Ref. 5 for details).

Only the last term in this expressions is caused by the quantum nature of the emitted gravitons. In fact, Eq. (3.7) agrees with the classical result for the emitted power by inserting an energy per graviton $\hbar \omega_{i f}$ into the sum and replacing $\omega_{i f}^{2}$ times the square of the matrix elements by the classical expression $\left(M^{-2} \partial_{t} q_{k l}\right)^{2} / 2$. Recall that according to the correspondence principle, we find the quantum-mechanical result for the transition $|i\rangle$ to $|f\rangle$ from the classical formula by replacing $q_{i j}(t) \rightarrow\left\langle f\left|\hat{q}_{i j}\right| i\right\rangle e^{-i \omega_{i f} t}+$ c.c. $=2\left|\left\langle f\left|\hat{q}_{i j}\right| i\right\rangle\right| \cos \left(\omega_{i f} t-\delta\right)$ from which we deduces the replacement of the classical $\left(\partial_{t} q_{i j}\right)^{2}$ by the temporal average of the quantum-mechanical $2 \omega_{i f}^{2}\left|\left\langle f\left|\hat{q}_{i j}\right| i\right\rangle\right|^{2}$.

A possible future application of our master equation (3.3) could be to neutrino oscillation experiments, modifying the discussions of Refs. [8,9].

\section{LANGEVIN EQUATION}

At high and moderately high temperatures, the fluctuations of $\mathbf{y}$ are small due to the last term in the exponent of (2.8). We therefore introduce average and difference variables of the forward and backward paths in (2.8):

$$
\mathbf{x} \equiv\left(\mathbf{x}_{+}+\mathbf{x}_{-}\right) / 2, \quad \mathbf{y}=\mathbf{x}_{+}-\mathbf{x}_{-},
$$

and expand $V\left(\mathbf{x}_{ \pm}\right)=V(\mathbf{x} \pm \mathbf{y} / 2)$ in $\mathbf{y}$ up to the third order. Then the exponent in Eq. (2.8) becomes

$$
\begin{aligned}
\frac{i}{\hbar} \int_{t_{a}}^{t_{b}} d t\left\{M \dot{\mathbf{y}} \dot{\mathbf{x}}-\mathbf{y} \nabla V(\mathbf{x})-\frac{1}{24} y_{i} y_{j} y_{k} \nabla_{i} \nabla_{j} \nabla_{k} V(\mathbf{x})-2 \frac{\gamma \hbar}{c^{4}}\left[\dot{\mathbf{x}}^{2}(\ddot{\mathbf{x}} \dot{\mathbf{y}})+\frac{1}{3}(\dot{\mathbf{y}} \dot{\mathbf{x}})(\ddot{\mathbf{x}} \dot{\mathbf{x}})+\frac{1}{3}(\ddot{\mathbf{y}} \dot{\mathbf{y}})(\dot{\mathbf{x}} \dot{\mathbf{y}})+\frac{1}{4} \dot{\mathbf{y}}^{2}(\ddot{\mathbf{y}} \dot{\mathbf{x}})\right]\right. \\
\left.+2 i \frac{w \hbar}{c^{4}}\left[\dot{\mathbf{y}}^{2} \dot{\mathbf{x}}^{2}+\frac{1}{3}(\dot{\mathbf{y}} \dot{\mathbf{x}})^{2}\right]\right\},
\end{aligned}
$$

where fourth and higher orders in $\mathbf{y}(t)$ are neglected. Neglecting also the quadratic terms $\mathbf{y}(t)$, extremization with respect of $\mathbf{y}(t)$ gives the classical equation of motion with radiation damping

$$
M \ddot{\mathbf{x}}+\nabla V-2 \frac{\gamma \hbar}{c^{4}} \frac{d}{d t}\left[\ddot{\mathrm{x}} \dot{\mathrm{x}}^{2}+\frac{1}{3} \dot{\mathrm{x}}(\ddot{\mathrm{x}} \dot{\mathrm{x}})\right]=0
$$

From this equation we can calculate the energy dissipation of the gravitational body due to the coupling to the bath of gravitons:

$$
\frac{d}{d t}\left\{\left[\frac{M \dot{\mathbf{x}}^{2}}{2}+V(\mathbf{x})\right]-\frac{2}{3} \frac{\gamma \hbar}{c^{4}} \frac{d}{d t}\left(\dot{\mathbf{x}}^{2}\right)^{2}\right\}=-2 \frac{\gamma \hbar}{c^{4}}\left[\ddot{\mathbf{x}}^{2} \dot{\mathbf{x}}^{2}+\frac{1}{3}(\ddot{\mathbf{x}} \dot{\mathbf{x}})^{2}\right] .
$$

Expressed in terms of $q_{k l}$, the right-hand side yields a rate of energy loss

$$
\dot{E}=-\gamma \hbar \dot{q}_{k l}^{2}=\frac{4 G}{5} \dot{q}_{k l}^{2}
$$

This is the formula derived for classical gravitational radiation. The extra term in the brackets is the gravitational analog of Schott's term in the energy balance of electromagnetic radiation damping discussed in [5, 10, 11].

Interpreting this equation twice over $t$ we can conclude that the time-average of the energy decreases owing to the dissipative term on the r.h.s. of (4.4). The quadratic fluctuations of $\mathbf{y}(t)$ in the path integral (2.10) are governed by the quadratic exponent (4.2), which can be written as a path integral

$$
\int \mathcal{D} \mathbf{x} \mathcal{D} \mathbf{y} \exp \left\{i \int_{t_{a}}^{t_{b}} d t\left[-\frac{\mathcal{M}}{2} \dot{y}_{k} \Omega_{k l} \dot{y}_{l}-\frac{i}{\hbar} y_{k} \dot{u}_{k}\right]\right\} F(\mathbf{x})
$$

where $\dot{\mathbf{u}}(t)$ abbreviates the left-hand side of Eq. (4.3),

$$
\mathcal{M} \equiv \frac{4 w}{3 c^{4}}, \quad \Omega_{k l} \equiv \dot{x}_{k} \dot{x}_{l}+3 \dot{\mathbf{x}}^{2} \delta_{k l} .
$$

The exponent can be completed quadratically to

$$
\int_{t_{a}}^{t_{b}} d t\left\{-\frac{\mathcal{M}}{2}\left[\left(\dot{y}_{k}-\frac{i}{\mathcal{M} \hbar} \Omega_{k l}^{-1} u_{l}\right) \Omega_{k r}\left(\dot{y}_{r}-\frac{i}{\mathcal{M} \hbar} \Omega_{r l}^{-1} u_{l}\right)\right]-\frac{1}{2 \mathcal{M} \hbar^{2}} u_{l} \Omega_{l r}^{-1} u_{r}\right\} .
$$


We now introduce the stochastic variable

$$
\eta \equiv \Omega^{-1 / 2} \mathbf{u}
$$

which has the white-noise correlation function

$$
\left\langle\eta_{i}(t) \eta_{j}\left(t^{\prime}\right)\right\rangle=\mathcal{M} \hbar^{2} \delta_{i j} \delta\left(t-t^{\prime}\right)=\frac{4 w \hbar^{2}}{3 c^{4}} \delta_{i j} \delta\left(t-t^{\prime}\right) .
$$

Ignoring the Jacobian of the transformation $\mathbf{x}(t) \rightarrow \mathbf{u}(t)$, the path integral is seen to be equivalent to the Langevin equation

$$
M \ddot{\mathbf{x}}+\nabla V(\mathbf{x})-2 \frac{\gamma \hbar}{c^{4}} \frac{d}{d t}\left[\ddot{\mathbf{x}} \dot{\mathbf{x}}^{2}+\frac{1}{3} \dot{\mathbf{x}}(\ddot{\mathbf{x}} \dot{\mathbf{x}})^{2}\right]=\frac{d}{d t} \Omega^{1 / 2} \boldsymbol{\eta} .
$$

Note that this Langevin equation is purely classical, since the constants $\gamma \hbar$ and $w \hbar^{2}$ are $\hbar$-independent. In electromagnetism, a similar equation has been derived in Ref. [5] solved in Ref. [12].

The omitted Jacobian consists of a product of two Jacobians for the transformations $\mathbf{x} \rightarrow \dot{\mathbf{u}}$ and $\dot{\mathbf{u}} \rightarrow \boldsymbol{\eta}$. For the latter, it is equal to Det $\partial_{t} \Omega^{1 / 2}$ which corresponds to an effective action $\log \operatorname{Det} \partial_{t} \Omega^{1 / 2}$ proportional to $\delta(0)$, This vanishes in dimensional regularization [4. The other Jacobian is nontrivial and awaits a proper evaluation along the lines of Ref. [13], and the result may modify Eq. (4.11).

The equation is applicable only for high temperatures. If we want to apply it to moderately high temperatures, quantum effects appear. To lowest order in $\hbar$, these arise from the first nonleading Taylor expansion term of $\operatorname{coth}\left[\beta \hbar \Omega_{\mathbf{k}} / 2\right]$ in (2.4). This term changes the $\delta$-function in $A_{k l ; m n}$ of Eq. (2.11) to

$$
\delta\left(t-t^{\prime}\right) \rightarrow \delta\left(t-t^{\prime}\right)-\frac{\hbar^{2} \beta^{2}}{24} \ddot{\delta}\left(t-t^{\prime}\right) .
$$

This leads to a change $\boldsymbol{\eta} \rightarrow \boldsymbol{\eta}-\hbar^{2} \beta^{2} \ddot{\boldsymbol{\eta}} / 24$ on the right-hand side of the Langevin equation (4.11).

Let us point out that our derivation of the classical equation (4.4) in the present quantum mechanical context is much simpler and more direct than the purely classical derivations in the literature. There one always start from extended objects to find the forces exerted upon a body by its own gravitational radiation. The subsequent limit of a point particle is quite delicate. For reviews on the subject see Refs. 10, 14, 15.

This research is supported by a grant from a governmental German university support program HSP III - Potsdam.

[1] R.P. Feynman and F.L. Vernon, Ann. Phys. (N.Y.) 24, 118 (1963)

[2] R.P. Feynman, A.R. Hibbs, Quantum Mechanics and Path Integrals, McGraw Hill, New York 1965

[3] A.O. Caldeira and A.J. Leggett, Ann. Phys. 149, 374 (1983), 153, 445(E) (1984).

[4] H. Kleinert, Path Integrals and Quantum Mechanics, Statistics, and Polymer Physics, 2nd edition, World Scientific, Singapore, 1995.

[5] Z. Haba, H. Kleinert, Master equation for electromagnetic dissipation and decoherence of density matrix, Berlin preprint 2000

[6] A discussion of the x-dependence can be found in Z. Haba, Mod. Phys. Lett. A 15, 1519 (2000).

[7] G. Lindblad, Comm. Math. Phys. 48, 119 (1976).

[8] S. Adler, Phys. Rev. D 62, 117901 (2000).

[9] E. Lisi, A. Marrone, and D. Montanino (hep-ph/0002053).

[10] F. Rohrlich, Classical and Charged Particles, Addison-Wesley, Reading. MA, 1963. Phys. Rev. D 60, 084017 (1999); Am. J. Phys. 68, 1109 (2000),

[11] F. Rohrlich, Phys. Rev. D 60, 084017 (1999); Am. J. Phys. 68, 1109 (2000),

[12] Z. Haba, H. Kleinert, Langevin Equation for Particle in Thermal Photon Bath, Berlin preprint 2000

[13] For more details on dimensional regularization in the present context see H. Kleinert, Ann. Phys. (N.Y.) (in press) quant$\mathrm{ph} / 0008109$ ).

14] Y. Mino, M. Sasaki, and T. Tanaka, Phys. Rev. D 55, 3457 (1997).

[15] T.C. Quinn and R.M. Wald, Phys. Rev. D 56, 3381 (1997). 